Check for updates

Cite this: RSC Adv., 2018, 8, 22506

\title{
Improvement in physical properties of single-layer gas diffusion layers using graphene for proton exchange membrane fuel cells
}

\author{
Hung-Fan Lee, ${ }^{a}$ Jing-Yue Chang ${ }^{a}$ and Yui Whei Chen-Yang (D) *ab
}

Gas diffusion layer (GDL) is an important component related to the efficiency of proton exchange membrane fuel cells (PEMFCS). Nevertheless, the preparation cost of the conventional GDL is high. In our previous studies, a single-layer gas diffusion layer (SL-GDL) prepared by a simple and cost-effective process has been used for PEMFCs, and it achieved $85 \%$ efficiency of a commonly used commercial GDL. In this study, improvement in physical properties of a series of single-layer gas diffusion layers, SLGDL-Gx $(x=1-3)$, via uniform distribution of graphene in the SL-GDL, and the application of SL-GDL-Gx in PEMFCs are studied. The results indicate that the presence of well-distributed graphene layers in SLGDL-Gxs causes an increase in the surface roughness and the formation of irregular slender interstices, leading to the enhancement of gas permeability while maintaining the microporous layer (MPL)-like microstructure and retaining good loading and efficient utilization of the catalyst. Moreover, the electrical resistivities significantly decreased and the mechanical properties improved. These improvements in physical properties are significantly beneficial for the performance of PEMFC. The single-cell performance tests show that the best performance measured at $80{ }^{\circ} \mathrm{C}$ under $99.9 \%$ relative humidity $(\mathrm{RH})$ conditions is obtained from the PEMFC (FC-2) fabricated with SL-GDL-G2 and is $46 \%$ higher than that from FC-0 with SL-GDL-G0 without graphene and $15 \%$ higher than that from FC-3 with the commercial GDL. Furthermore, the performances of FC-2 measured at $50-80{ }^{\circ} \mathrm{C}$ under $15 \% \mathrm{RH}$ are all much higher than those of FC-3. The results indicate that SL-GDL-G2 prepared via a cost-effective method is a potential GDL for PEMFCs.

Received 8th March 2018 Accepted 2nd June 2018

DOI: $10.1039 / \mathrm{c} 8 \mathrm{ra02062k}$

rsc.li/rsc-advances

\section{Introduction}

PEMFC is one of the promising alternative and clean energy source for a variety of applications due to the advantages, such as high energy conversion efficiency, low emission, low noise, and fast start-up. ${ }^{\mathbf{1 - 4}}$ It is known that both proton exchange membranes (PEMs) and gas diffusion electrodes (GDEs) are core components in the membrane electrode assembly (MEA) and the important in determining the efficiency of PEMFCs. ${ }^{5,6}$ In general, the efficiency of PEMFCs with the commercial MEA is usually good at low temperatures under both high and low relative humidity, while it is poor as the temperature increases or the RH decreases because the elevated temperature and the lowered $\mathrm{RH}$ results in dehydration in MEA, thus reducing the efficiency.

\footnotetext{
${ }^{a}$ Department of Chemistry, Chung Yuan Christian University, 200 Chung Pei Road, Chung Li District, Taoyuan City, Taiwan 32023, Republic of China

${ }^{b}$ Center for Nanotechnology and Center for Biomedical Technology, Chung Yuan Christian University, 200 Chung Pei Road, Chung Li District, Taoyuan City, Taiwan 32023, Republic of China. E-mail: yuiwhei@cycu.edu.tw; Fax: +886 3265 3399; Tel: +88632653317
}

In order to mitigate the dehydration of MEA, some studies focused on the modification of PEM. ${ }^{7-10}$ Nevertheless, the dehydration condition of PEM is also influenced by the structure of GDLs, a critical part in GDE, used. ${ }^{11-13}$ The GDLs function as channels for fuel gas transport, routes for electron conduction, and regulators for water management, ${ }^{\mathbf{5 1 4 - 2 0}}$ which are related to the gas permeability, electrical conductivity, and hydrophobicity, respectively, thus influencing the performance of PEMFCs. Nevertheless, the preparation process of the conventional GDL requires high-temperature heat treatment and numerous complicated steps, which increases the cost. ${ }^{\mathbf{5} 21}$ Therefore, the development of new GDLs to reduce the cost and enhance the performance efficiency is necessary. In our previous study, the single-layer gas diffusion layer (SL-GDL) with a MPL-like microstructure was prepared. ${ }^{18}$ Unlike the traditional dual-layer GDL, which is formed with MPL and macroporous gas diffusion medium (GDM), the single-layer SLGDL without GDM was prepared by a simple and less expensive method. It was found that on application to PEMFCs, the efficiency of SL-GDL already reached $85 \%$ that of the commercial dual-layer GDL.

Recently, graphene has emerged as an important and exciting material. It has attracted the attention of both 
theoretical and experimental researchers and has been widely used in numerous applications owing to its unique properties. $^{22-25}$ Graphene has also been introduced into the MPL as a cross-linker between the carbon xerogels ${ }^{26}$ and directly mixed with carbon powder $^{27}$ to improve the electrical conductivity, pore structure, and the related physical properties, thus enhancing the performance of PEMFCs even at low relative humidity. ${ }^{26,27}$ However, only few studies have reported the influence of graphene on the properties of GDLs.

In this study, graphene was used as an additional filler to prepare a series of graphene-containing single-layer gas diffusion layers, SL-GDL-G $x(x=1-3)$, in order to investigate its influence on the physical properties. Because of the intrinsic properties of graphene, it is anticipated that not only lower electrical resistivity and improved mechanical properties will be obtained, but also larger average pore size and rougher surface can be achieved, which in turn will increase the efficiency, which can be competed with that of the commercial dual-layer GDL for PEMFCs.

\section{Experimental}

\subsection{Preparation of graphene-containing single-layer gas diffusion layers (SL-GDL-Gxs)}

A series of SL-GDL-G $x$ s $(x=0-3)$ were prepared by adding graphene (CG-003, Conjutek) into SL-GDL-CF15, which was prepared with carbon black (Vulcan XC-72, Cabot Co.), VGCFs (T-004, Yonyu Applied Technology Material Co., Ltd., Taiwan), and colloidal PTFE dispersion (D1-E, Daikin Industries Ltd.) according to the method reported previously. ${ }^{18}$ The graphene used in this study was an average of 4-5 layers with approximately 10-25 $\mu \mathrm{m}$ width. Initially, VGCFs, carbon black, and graphene were homogeneously mixed with the aid of an ultrasonicator (Q700, Qsonica) and a homogenizer (HD-0025RDM, Shin-Kwang Precision Industry Ltd.) in sufficient amount of ethyl alcohol. The PTFE dispersion was then added to the mixture and mixed well for few minutes using a mechanical stirrer equipped with a heater and then, the ethanol was removed. Subsequently, the mixture was pressed by a homemade calender at room temperature and then compressed under $75 \mathrm{~kg} \mathrm{~cm}^{-2}$ at $80{ }^{\circ} \mathrm{C}$ into a film of $0.38 \pm 0.02 \mathrm{~mm}$ average thickness. Finally, the film was sintered at $130{ }^{\circ} \mathrm{C}$ for $2 \mathrm{~h}$ and cooled to room temperature to obtain the SL-GDL-Gxs thin film. The corresponding compositions of the SL-GDL-Gxs are listed in Table 1. For comparison, the commercial GDL was obtained from SIGRACET®, Germany.

\subsection{Characterization of surface morphology and physical properties of GDLs}

The surface morphologies of SL-GDL-Gxs were examined using a field-emission scanning electron microscope (FESEM, JSM7600 , JOEL). The specimens were used without coating a layer of platinum because of the intrinsically high electrical conductivity of the GDLs. The surface roughness of SL-GDL was examined using an atomic force microscope (AFM, XE100, Korea). The porosity, average pore diameter, and pore size
Table 1 The compositions of the SL-GDL-Gxs ${ }^{a}$

\begin{tabular}{llll}
\hline & \multicolumn{2}{l}{ Ingredients } & \\
\cline { 2 - 4 } & $\begin{array}{l}\text { Carbon black }+ \\
\text { VGCF (wt\%) })^{*}, b\end{array}$ & $\begin{array}{l}\text { Graphene } \\
(\mathrm{wt} \%)^{b}\end{array}$ & $\begin{array}{l}\text { PTFE content } \\
(\mathrm{wt} \%)^{c}\end{array}$ \\
\hline SL-GDL-G0* & 100.0 & 0.0 & 10 \\
SL-GDL-G1 & 97.5 & 2.5 & 10 \\
SL-GDL-G2 & 95.0 & 5.0 & 10 \\
SL-GDL-G3 & 90.0 & 10.0 & 10
\end{tabular}

$a *$ The same as SL-GDL-CF15 published previously, ${ }^{18 * *}$ the weight ratio of carbon black : VGCF ( $85: 15)$ is the same as that in SL-GDL-CF15. ${ }^{b}$ The weight $\%$ based on the total weight of all the carbon materials, including carbon black, VGCF, and graphene. ${ }^{c}$ The weight $\%$ based on the total weight of the whole material.

distribution were measured by mercury intrusion porosimetry using Auto pore 9220, Micromeritics.

The physical properties such as electrical resistivity and gas permeability, and mechanical properties of GDLs were characterized as follows: the through-plane electrical resistance under $3 \mathrm{MPa}$ and the in-plane electrical resistivity of the GDL were measured by the two-electrode and four-point probe methods using the combined system of a potentiostat (PGSTAT30, Metrohm Autolab B.V.) and a current voltmeter (Keithley 2400), respectively.

The gas permeabilities of the SL-GDL-Gxs were determined using a gas permeability analyzer designed as reported. ${ }^{28}$ The mechanical properties of the SL-GDL-Gxs were determined using a tensile tester (Shimadzu EZ test) equipped with a $500 \mathrm{~N}$ load cell and a computer interface for data collection. All samples were prepared according to ASTM D638.

\subsection{Fabrication of MEA and cell performance test}

In order to rule off the effect from the other parameters and focus on studying the effect on the performance of PEMFCs caused by using different GDEs, all the GDEs were prepared following the same procedure from different GDLs, including the as-prepared SL-GDL-Gxs and SGL 35BC. The single-cell tests were all performed using our home-made FC system under the same operating condition. The MEAs for this study were fabricated using a Nafion ${ }^{\circledR}$ membrane (NRE 212) and the CL-coated GDLs. The PEMFCs with SL-GDL-G0, SL-GDL-G1, SL-GDL-G2, and SGL 35BC are named as FC-0, FC-1, FC-2, and FC-3, respectively.

The MEAs with an active area of $5 \mathrm{~cm}^{2}$ were fabricated via a hot-pressed procedure at $140{ }^{\circ} \mathrm{C}$ under $30 \mathrm{~kg} \mathrm{~cm}{ }^{-2}$ for 3 minutes. ${ }^{2}$ Prior to the hot-pressing procedure, all GDEs were prepared following the same procedure to rule off the preparation effect. Each GDL was coated with a catalyst layer (CL) to obtain the corresponding GDE for use in the cell test. The CL was prepared as follows. Initially, $20 \mathrm{wt} \%$ of Pt/C (HiSpec 3000, Johnson Matthey) was dispersed in ethyl alcohol with ultrasonication to obtain a catalyst slurry. Then, the slurry was sprayed onto the GDL surface using a spray coater (Hephas Energy Co., Ltd). The loading amounts of Pt used for both the 
anode and the cathode were $0.4 \mathrm{mg} \mathrm{cm}^{-2}$, as measured by ICPMS (Agilent 7500ce, Japan) in all GDEs. The electrolyte membrane used was a Nafion ${ }^{\circledR}$ membrane of DuPont Company (NRE 212). This membrane was pretreated prior to use according to an established membrane cleaning procedure. ${ }^{2}$

The single-cell fixture was composed of the as-prepared MEA and a pair of graphite plates with a serpentine flow channel of $1 \mathrm{~mm}$ width and $1 \mathrm{~mm}$ depth. In order to prevent the GDL from getting into the channels, the MEA was placed between a pair of PTFE gaskets with thickness of $0.1 \mathrm{~mm}$ each. It was clamped between two stainless end plates, with eight bolts tightened to a uniform torque of $2.94 \mathrm{~N}$ m to ensure good contact of GDLs with the graphite plates and prevent the GDL from getting into the channels.

For the single-cell test, the inlet fuel gases of the anode and the cathode were hydrogen and oxygen, respectively. The test was carried out under atmospheric pressure. The relative humidity $(\mathrm{RH})$ values of the inlet gases measured immediately after passing through the external bubble-type humidifier at $50{ }^{\circ} \mathrm{C}$ were approximately $99.9 \%$ and $15 \%$ when no humidification was applied. The fuel gas flow rate of the anode and the cathode were 70 and $102 \mathrm{~mL} \mathrm{~min}^{-1}$, respectively. The cell test was performed at cell temperatures $\left(T_{\text {cell }}\right)$ of $50,60,70$ and $80{ }^{\circ} \mathrm{C}$ without changing the humidification temperature $\left(50{ }^{\circ} \mathrm{C}\right)$. Before the cell test, a leak test was performed and a constant current was provided to the single cell to activate the MEA until stable current density was obtained., ${ }^{2,18}$ The test proceeded under atmospheric pressure, and the cell efficiency was obtained by controlling the cell voltage and subsequently recording the corresponding stabilized current. The electronic load was controlled using the software, NOVA version 1.3, and interfaced with a computer for data collection.

\section{Results and discussion}

\subsection{The effect of various amounts of graphene on surface morphologies of SL-GDL-Gxs}

The SEM micrograph of the top-view images of pristine graphene used in this study shows a paper-like morphology and a smooth surface as expected. Fig. 1(a)-(e) displays the SEM images of the top-view of SL-GDL-G $x$ s and the MPL side of SGL $35 \mathrm{BC}$ at low magnification. A relatively smooth surface for SLGDL-G0 without graphene is shown in Fig. 1(a). Moreover, no distinct aggregation of graphene layers is found, as shown in Fig. 1(b) and (c), indicating that the graphene layers were well mixed in SL-GDL-G1 and SL-GDL-G2 by the homogenizing process. However, as indicated in Fig. 1(d), when the graphene content reached $10 \mathrm{wt} \%$ in SL-GDL-G3, some agglomerates are observed (indicated by red circles). Moreover, it is seen that as the amount of graphene was increased, lumps were gradually formed and overlapped with one another to form the micrometer-scale irregular slender interstices (indicated by arrows in Fig. 1(c)), resulting in an increase in the surface roughness. The surface roughness $\left(R_{\mathrm{a}}\right)$ values of SL-GDL-G0, SLGDL-G1, and SL-GDL-G2 measured by AFM are $0.365,0.824$, and 1.028, respectively. It is believed that the interstices would facilitate gas permeation when GDLs are used in the PEMFC performance test as reported. ${ }^{29}$ Furthermore, Fig. 1(e) shows a smooth surface for the MPL-side of the commercial GDL, SGL 35BC, as expected.

In Fig. 1(f)-(j), the highly magnified SEM images of the topviews of SL-GDL-Gxs and the MPL side of SGL 35BC show that although the degrees of their surface roughness were different as observed in the low-magnification images (Fig. 1(a)-(e)), Fig. 1(g)-(i) exhibit similar microstructures for the graphenecontaining SL-GDL-G $x$ s as that for the graphene-free SL-GDLG0 (Fig. 1(f)) and the MPL side of SGL 35BC (Fig. 1(j)), i.e., MPL-like microstructures. These results reveal that the presence of graphene in SL-GDL-Gxs caused an increase in the surface roughness and the formation of micrometer-scale interstices that would enhance the gas permeability while maintaining the MPL-like microstructure surfaces that ensured good loading and efficient utilization of the catalyst.

The average pore diameters and the pore size distribution of the SL-GDL-Gxs measured using a mercury porosimeter are listed in Table 2 and shown in Fig. 2. The average pore diameter of SL-GDL-GxS was much smaller than that of SGL 35BC and it increased with the increase in the amount of graphene added (up to SL-GDL-G2). The two peaks near $60 \mathrm{~nm}$ and $50 \mu \mathrm{m}$ for SGL 35BC are attributed to the intrinsic property of MPL and carbon paper, respectively. As listed in Table 2, the average pore diameter of SL-GDL-G2 was about 4.16 times larger than that of SL-GDL-G0. This is attributed to the formation of the interstices as shown in the SEM images. However, as shown in Fig. 2, similar to that of MPL in SGL 35BC, the sizes of the pores in SLGDL-G0 and SL-GDL-G1 are centered at around $25 \mathrm{~nm}$ and $60 \mathrm{~nm}$, while the pore size of SL-GDL-G2 was over $50 \mathrm{~nm}$, indicating that the pore structure was changed and the pores were enlarged due to the addition of graphene, which were anticipated to improve the gas permeability and the bi-phase dynamic flow. A similar result was reported for the GDL composites reported by Han et al., in which the enlarged pore size was also attributed to the addition of graphite. ${ }^{29}$

\subsection{Physical properties of SL-GDL-Gxs}

The through-plane electrical resistances and in-plane electrical resistivities of the SL-GDL-G $x$ s and SGL 35BC are listed in Table 3. Although the electrical resistances and resistivities of SLGDL-Gxs were all higher than those of SGL 35BC, they were lowered with the addition of graphene. This is mainly ascribed to the additional electron transport bridges provided by the paper-like graphene, which interconnected and covered the disjointed carbon black particles to shorten the transport path of electrons, as illustrated in Fig. 3. However, as the content of graphene increased to $10 \mathrm{wt} \%$, i.e., in case of SL-GDL-G3, the electrical resistivities did not decreased further, but slightly increased due to the slight agglomeration and rougher surface, causing poorer interfacial contact with the electrodes. This implies that apart from the intrinsic resistivity, the electrical resistivity of the GDL was also affected by the degree of interfacial contact between the GDL and the electrode.

Moreover, because the GDL in PEMFC is sandwiched between the CL and the gas-flow channel, GDL applicable for 

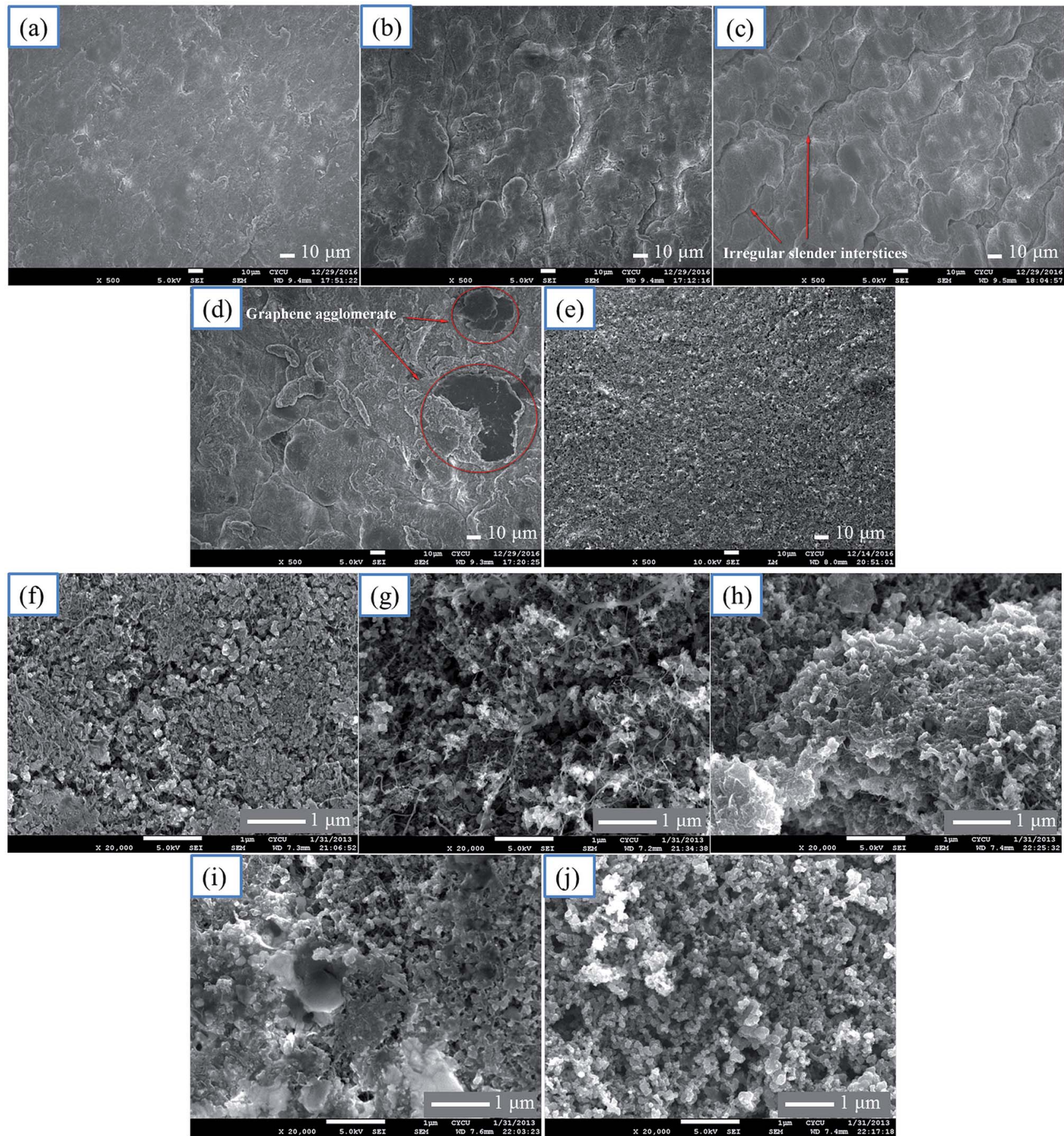

Fig. 1 SEM images of top-view of SL-GDL-Gx: (a) $x=0$, (b) $x=1$, (c) $x=2$, (d) $x=3$, and (e) MPL side of SGL 35BC at 500 $\times$ magnification, and (f) $x$ $=0$, (g) $x=1$, (h) $x=2$, (i) $x=3$, and (j) MPL side of SGL 35BC at $20000 \times$ magnification.

Table 2 Mechanical properties and average pore diameters of the GDLs

\begin{tabular}{llllll}
\hline Sample & $\begin{array}{l}\text { Yield stress } \\
(\mathrm{MPa})\end{array}$ & $\begin{array}{l}\text { Yield elongation } \\
(\%)\end{array}$ & $\begin{array}{l}\text { Tensile strength } \\
(\mathrm{MPa})\end{array}$ & $\begin{array}{l}\text { Elongation at } \\
\text { break }(\%)\end{array}$ & $\begin{array}{l}\text { Porosity } \\
(\%)\end{array}$ \\
\hline SL-GDL-G0 & 0.18 & 5.6 & 0.23 & 8.70 & 79 \\
SL-GDL-G1 & 0.33 & 20.4 & 0.37 & 23.80 & 71 \\
diameter $(\mu \mathrm{m})$
\end{tabular}




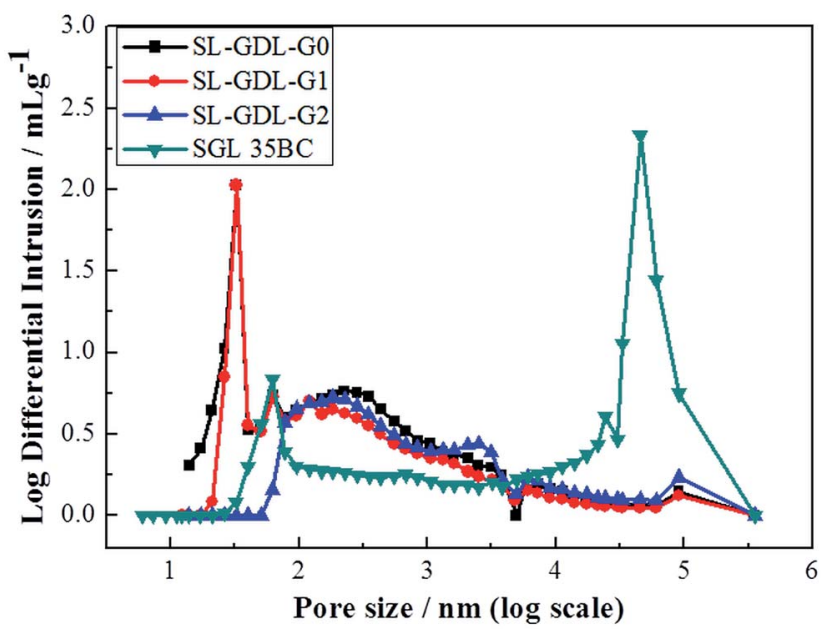

Fig. 2 Pore size distribution of SL-GDL-Gxs and SGL 35BC measured using a mercury intrusion porosimetry.

PEMFC has to be neither very stiff nor very soft, so that it can simultaneously support and establish good contact with the CL and also, it should not break or intrude into the gas-flow channel under pressure. Thus, the mechanical property has to be good enough to prevent deformation and maintain structural integrity during the performance test. ${ }^{30}$ Table 2 indicates that compared with that of SL-GDL-G0, the yield stress of the SLGDL-G1 increased from 0.18 MPa to $0.33 \mathrm{MPa}, v i z$, about $83 \%$ improvement, due to the addition of graphene, which is known to have very high mechanical strength. ${ }^{31}$ This improvement is favorable for the preparation of the membrane electrode assembly and the pressure tolerance. The slight deviation in yield elongation for SL-GDL-G1 and SL-GDL-G2 is attributed to the difference in the loading amounts and the corresponding distribution densities of graphene. The high yield stress and small yield elongation of SGL 35BC were attributed to the mechanically strong carbon paper GDM. Although the SL-GDLGxs were not as strong as that of SGL 35BC, no deformation of SL-GDL-G $x$ s was observed and structural integrity was maintained after the single-cell performance test and the measurement of through-plane electrical resistance under $3 \mathrm{MPa}$. This affirmed that the SL-GDL-Gxs are applicable in PEMFCs.

Homogeneous and efficient transport of fuel gasses from the GDLs to the catalyst surface is one of the crucial requirements for an ideal GDL during the operation of PEMFCs. Typically, the current is influenced by the kinetics of the reaction in a lowcurrent-density region, while mass transport is often related to the diffusion of the reactants in a high-current-density region. ${ }^{16,21}$ Therefore, the gas permeability in GDL is essential for studying the mass transport in fuel cells. As listed in Table 3, the gas permeability of SL-GDL-G2 was about $215 \%$ and $226 \%$ higher than that of SL-GDL-G1 and SL-GDL-G0, respectively. This is ascribed to the existence of the interstices in SL-GDL-G2, thus facilitating the gas transport. In case of SL-GDL-G3, the decrease in gas permeability is attributed to the aggregation of graphene in the GDL that might hinder the gas transport. The highest value of gas permeability for SGL 35BC was attributed to the existence of the largest average pore size. Since the electrical resistivity and gas permeability of SL-GDL-G3 studied above were all poor than that of SL-GDL-G2, SL-GDL-G3 was not investigated further in this study.

In summary, the physical properties of SL-GDL indeed improved by the addition of graphene. Among the as-prepared SL-GDLs, SL-GDL-G2 exhibited a better combination of electrical resistivity, air permeability, and mechanical property. Therefore, the PEMFC with SL-GDL-G2 was expected to be superior to those with other SL-GDLs.

\subsection{Single cell performance test of PEMFCs}

It is known that the performance efficiency of PEMFCs is related to the FC system used and the parameters applied. Each parameter used in the PEMFC such as the material of PEM, ${ }^{2,6}$ fuel gas flow rate, ${ }^{32}$ inlet air temperature and relative humidity, ${ }^{33}$ different humidifier systems, ${ }^{34}$ the type of flow field plate, ${ }^{35}$ humidification temperature and cell temperature, ${ }^{36}$ compression pressure and water management, ${ }^{37}$ and catalyst, ${ }^{38}$ can affect the performance.

To evaluate the efficiency of the as-prepared SL-GDL-Gxs on PEMFC performance, the single-cell test was carried out for FC-0, FC- 1 , and FC-2 at various cell temperatures $\left(50-80{ }^{\circ} \mathrm{C}\right)$ and $99.9 \% \mathrm{RH}$ of the inlet fuel gases at a temperature fixed at $50^{\circ} \mathrm{C}$ and compared with that of FC-3. The polarization curves and the power curves obtained are displayed in Fig. 4 and the data are listed in Table 4.

Table 4 indicates that the efficiencies of FC-3 measured by our home-made FC system were less than $720 \mathrm{~mW} \mathrm{~cm}{ }^{-2}$ and lower than those reported recently. ${ }^{39,40}$ Nevertheless, the results with the same FC system of our studies with different commercial GDEs gave similar performance efficiencies, ${ }^{2,41,42}$ indicating that although the efficiency was not as good as the others, ${ }^{39,40}$ our system works with consistency.

In general, under the same operating conditions of a FC system with different GDLs, as the cell temperature is changed, the PEMFC performance is determined by the physical property of the

Table 3 Electrical resistivity and air permeability of the GDLs

\begin{tabular}{lclc}
\hline Sample & $\begin{array}{l}\text { Through-plane electrical } \\
\text { resistivity }\left(\mathrm{m} \Omega \mathrm{cm}^{2}\right)\end{array}$ & $\begin{array}{l}\text { In-plane electrical } \\
\text { resistivity }(\Omega \mathrm{cm})\end{array}$ & $\begin{array}{l}\text { Air permeability } \\
\left(\mathrm{cm}^{3}(\mathrm{STP}) \mathrm{cm}^{\prime} / \mathrm{scm}^{2} \mathrm{~cm}_{-} \mathrm{Hg}\right)\end{array}$ \\
\hline SL-GDL-G0 & 12.9 & $1.44 \times 10^{-2}$ & $7.9 \times 10^{-3}$ \\
SL-GDL-G1 & 8.5 & $3.24 \times 10^{-3}$ & $7.5 \times 10^{-3}$ \\
SL-GDL-G2 & 9.1 & $3.71 \times 10^{-3}$ & $1.7 \times 10^{-2}$ \\
SL-GDL-G3 & 10.1 & $3.80 \times 10^{-3}$ & $1.0 \times 10^{-2}$ \\
SGL 35BC & 4.4 & $1.06 \times 10^{-3}$ & $1.0 \times 10^{-1}$
\end{tabular}




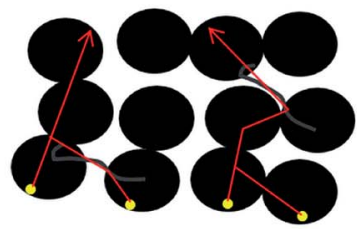

GDL without graphene

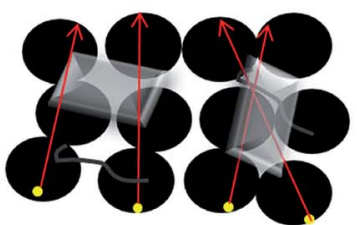

GDL with graphene

\section{$\chi$ carbon nanofiber \\ carbon black \\ graphene \\ electron}

$\longrightarrow$ route of electron

Fig. 3 Illustration of the electron transport of SL-GDL-Gxs with and without graphene.
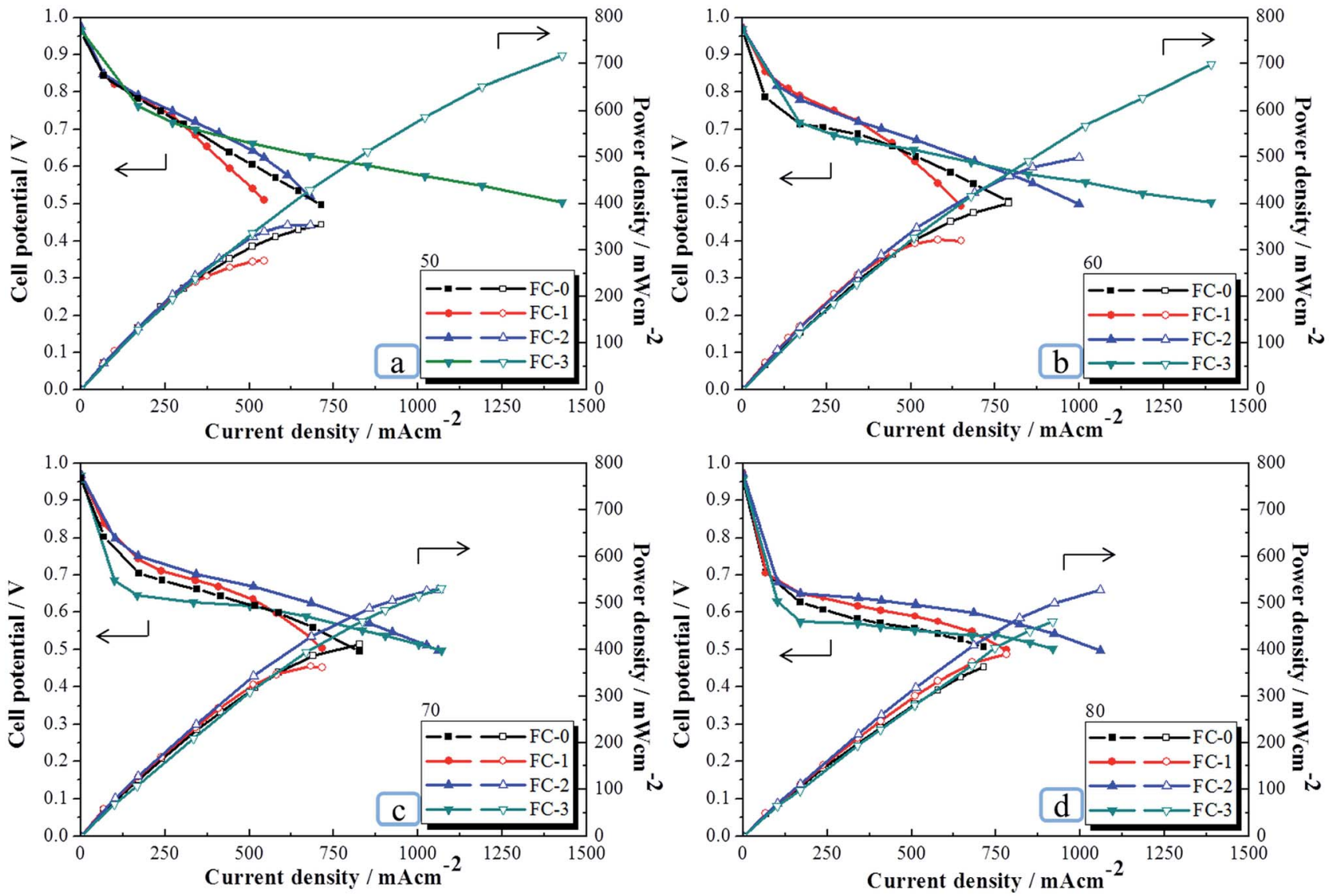

Fig. 4 Polarization curves ( $I-V$ curves) and $I-P$ curves of the PEMFC with SL-GDL-Gxs and SGL 35BC at different operating cell temperatures: (a) $50{ }^{\circ} \mathrm{C}$; (b) $60{ }^{\circ} \mathrm{C}$; (c) $70{ }^{\circ} \mathrm{C}$; and (d) $80^{\circ} \mathrm{C}$. The temperature and relative humidity of both the anode and the cathode were $50{ }^{\circ} \mathrm{C}$ and $99.9 \% \mathrm{RH}$, respectively.

GDL and the water management of MEA, which relates to the water absorption condition of PEM and the flooding condition of MEA. ${ }^{15,17}$

Table 4 Power densities obtained for the PEMFC at $0.5 \mathrm{~V}$ with various GDLs at a cell temperature between 50 and $80^{\circ} \mathrm{C}$ and under $99.9 \% \mathrm{RH}$

\begin{tabular}{lllll}
\hline & \multicolumn{4}{l}{ Power density $\left(\mathrm{mW} \mathrm{cm}^{-2}\right)$} \\
\cline { 2 - 5 }$T_{\text {cell }}\left({ }^{\circ} \mathrm{C}\right)$ & FC-0 & FC-1 & FC-2 & FC-3 \\
\hline 50 & 354.4 & 277.4 & 353.2 & 718.6 \\
60 & 400.4 & 320.4 & 498.9 & 698.9 \\
70 & 410.3 & 360.6 & 527.8 & 532.6 \\
80 & 361.5 & 390.2 & 527.4 & 460.5
\end{tabular}

As can be seen in Table 4, for FC-3, when the cell temperature was increased from $50{ }^{\circ} \mathrm{C}$ to $80{ }^{\circ} \mathrm{C}$, the performance efficiency gradually decreased for about $36 \%$ (from 718.6 to $460.5 \mathrm{~mW}$ $\mathrm{cm}^{-2}$ ). This is consistent with many reported results of the Nafion-based PEMFCs, ${ }^{2,36,43,44}$ and has been mainly attributed to the increase in dehydration of the Nafion PEM as elevating the cell temperature, resulting in the increase in membrane resistance. In particular, Pérez-Page et al. revealed that as the operation temperature was higher than the humidification temperature, the performance decreased accordingly because of the increase in the dryness (dehydration) of the membrane, resulting in decrease the membrane conductivity. ${ }^{36}$ Similarly, in this study, in spite of the RH of the inlet fuel gases being $99.9 \%$, 
since the humidification temperature was fixed at $50{ }^{\circ} \mathrm{C}$, the liquid/vapor two-phase water would reach the new equilibrium as the cell temperature increased from $50^{\circ} \mathrm{C}$ to $80^{\circ} \mathrm{C}$. Therefore, the amount of water absorbed in the PEM gradually decreased, leading to a decrease in the performance. This result revealed that the performance of FC-3 was dominated by the dehydration condition of the PEM under the condition measured due to the existence of the macropores, preventing the flooding condition.

The efficiencies of FC-3 were all better than that of SL-GDLbased FCs, including FC-0, FC-1, and FC-2, in the cell temperature range of $50-70{ }^{\circ} \mathrm{C}$. This is mainly ascribed to the better physical properties of SGL 35BC as discussed above and the absence of large pores in SL-GDL, which cause difficulty of draining out the liquid water, leading to a flooding condition, in this temperature range.

Nevertheless, contrary to the decreasing trend for FC-3, as the cell temperature was raised from $50{ }^{\circ} \mathrm{C}$ to $70{ }^{\circ} \mathrm{C}$, the power densities gradually increased by about $16 \%, 30 \%$ and $49 \%$ for FC-0, FC-1, and FC-2, respectively, due to the difference in the structures of SGL $35 \mathrm{BC}$ and SL-GDL-Gxs. It is believed that without the macropores in SL-GDL-Gxs, the flooding condition at the cathode was an inevitable factor at low temperature. In other words, unlike FC-3, the performances of the SL-GDLbased PEMFCs were affected by both the dehydration of PEM and the flooding condition of MEA in this cell temperature range. Nevertheless, as the cell temperature increased, the flooding condition gradually relieved, thus improving the performance. Furthermore, as mentioned above, the presence of graphene in SL-GDL-Gxs caused an increase in the surface roughness and the formation of interstices that could trap more water than SL-GDL-G0 and feed water back to the PEM, thus improving the water absorption capacity of PEM. Therefore, optimized performance would be obtained for each SL-GDLbased PEMFC at its best performance temperature, at which optimized balance of water absorption of PEM and relief from water flooding of MEA was achieved. Accordingly, the performances of FC-0, FC-1, and FC-2 were gradually improved as indicated in Table 4 . FC-0 showed the best performance at $70{ }^{\circ} \mathrm{C}$, while the graphene-containing FC- 1 and FC-2 showed the best performances at $80{ }^{\circ} \mathrm{C}$. Furthermore, the performance efficiencies of FC- 2 were all better than those of FC- 1 at the temperatures measured. This is ascribed to the larger average pore size and interstices in SL-GDL-G2 than in SL-GDL-G1, resulting in higher gas permeability and better water management in MEA for FC-2 than for FC-1. Due to the opposite trends of performance efficiencies for FC-2 and FC-3 with the increase in the temperature from 50 to $80{ }^{\circ} \mathrm{C}$, the power density for FC-2 $\left(527.8 \mathrm{~mW} \mathrm{~cm}^{-2}\right)$ reached $99 \%$ of that for FC-3 $\left(532.6 \mathrm{~mW} \mathrm{~cm}^{-2}\right)$ at $70{ }^{\circ} \mathrm{C}$ and was even $15 \%$ higher than that of FC-3 at $80{ }^{\circ} \mathrm{C}$, indicating better balance of kinetics and water
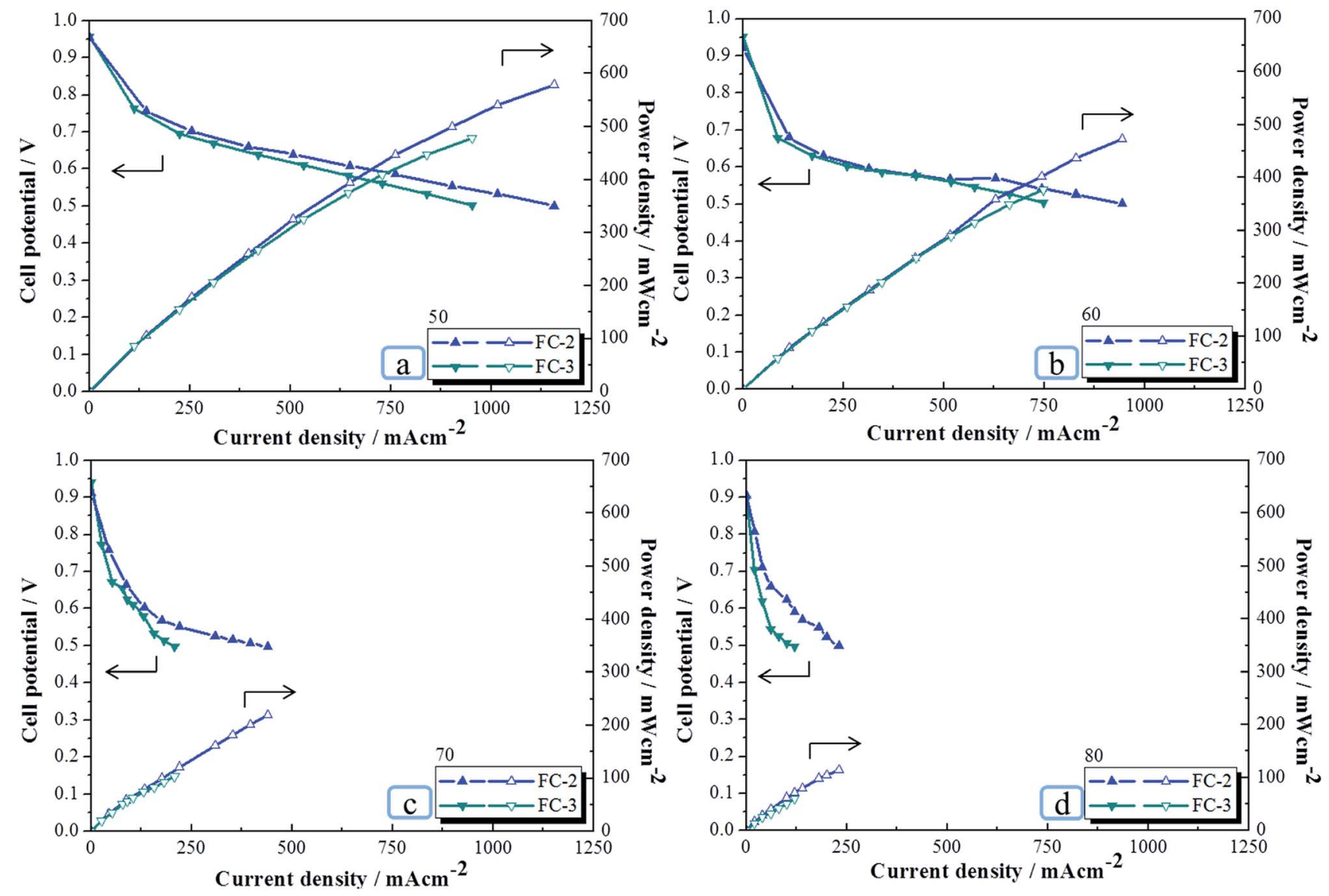

Fig. 5 Polarization curves ( $I-V$ curves) and I-P curves of the PEMFC with SL-GDL-G2 and SGL 35BC at different operating cell temperatures: (a) $50{ }^{\circ} \mathrm{C}$; (b) $60{ }^{\circ} \mathrm{C}$; (c) $70{ }^{\circ} \mathrm{C}$; and (d) $80{ }^{\circ} \mathrm{C}$. The $\mathrm{RH}$ is under approximately $15 \%$. 
Table 5 Power densities of FC-2 and FC-3 at various cell temperatures at $0.5 \mathrm{~V}$ and approximately $15 \%$ relative humidity

\begin{tabular}{llr}
\hline & \multicolumn{2}{l}{ Power density $\left(\mathrm{mW} \mathrm{cm}^{-2}\right)$} \\
\cline { 2 - 3 }$T_{\text {cell }}\left({ }^{\circ} \mathrm{C}\right)$ & FC-2 & FC-3 \\
\hline 50 & 578.7 & 478.5 \\
60 & 472.4 & 376.4 \\
70 & 219.2 & 103.7 \\
80 & 114.4 & 59.6 \\
\end{tabular}

management for FC-2 than for FC-3 at $80{ }^{\circ} \mathrm{C}$. These results indicate that SL-GDL-G2 is suitable for PEMFCs used at a medium operating cell temperature under $99.9 \% \mathrm{RH}$ of the inlet gases and has the potential for application in vehicles and grid-off system. .,4,5,8 $^{\mathbf{1}, \mathbf{n}}$

It is known that the FC system without any humidifier is simpler and lighter, in which no water flooding effect is observed. Therefore, the performances of FC-2 and FC-3 operating without humidifier $(15 \% \mathrm{RH})$ were also investigated. The polarization curves, $I-P$ curves, and power densities of FC-2 and FC-3 are shown in Fig. 5 and summarized in Table 5. As expected, the performances of both FC-2 and FC-3 decreased as the cell temperature increased due to the increase in the dehydration of PEM. Table 5 indicates that the power densities of FC-2 at $0.5 \mathrm{~V}$ are all higher than those of FC-3 and are approximately $21 \%, 26 \%, 111 \%$, and $113 \%$ higher than that of FC-3 at $50{ }^{\circ} \mathrm{C}, 60{ }^{\circ} \mathrm{C}, 70{ }^{\circ} \mathrm{C}$, and $80{ }^{\circ} \mathrm{C}$, respectively. This is attributed to the difference in the structures of the two GDLs. According to the above-discussed SEM images, it can be found that SL-GDL-G2 used in FC-2 has an MPL-like structure with irregularly distributed slender interstices that were able to trap water and feed water back to the PEM to maintain the mass transport, while the macropores in the GDM of FC-3 are too large to retain water inside the MEA under this low RH condition. Overall, the power densities of FC-2 were all higher than those of FC-3 in the temperature range of $50-80{ }^{\circ} \mathrm{C}$ because of the special structure and morphologies of SL-GDL-G2.

\section{Conclusion}

In this study, a series of graphene-containing single-layer gas diffusion layers, SL-GDL-Gxs, were successfully prepared by a simple and cost-effective method, and the physical properties were investigated. The SEM images showed that all the carbon materials, including carbon black, VGCFs and graphene, were well-distributed in the GDL and all the SL-GDL-Gxs exhibited MPL-like structure. Owing to the intrinsic properties of graphene, the physical properties of SL-GDL-Gxs were significantly improved. The electrical resistivity of the SL-GDL-G $x$ decreased, resulting in enhancement of the electron transportation, the mechanical strength was enhanced, bringing about the improvement in material integrity, and the micrometer-scale irregular slender interstices were formed, leading to an increase in the gas permeability and altering the pore structure. These improvements in the physical properties brought about the enhancement of the performance of PEMFCs. As a result, the power densities of FC-2 fabricated with SL-GDL-G2 at 99.9\% $\mathrm{RH}$ were all higher than those with SL-GDL-G0 as the temperature was over $60{ }^{\circ} \mathrm{C}$ and were about $15 \%$ higher than that of the PEMFC with SGL 35BC (FC-3) at $80{ }^{\circ} \mathrm{C}$. Moreover, at $15 \% \mathrm{RH}$, the power densities of FC-2 were all higher than those of FC-3 in the temperature range of $50-80{ }^{\circ} \mathrm{C}$. The graphene-containing SL-GDL-G2, prepared by a simple and cost-effective method, is anticipated to be a cost-effective, potential GDL for PEMFCs.

\section{Conflicts of interest}

There are no conflicts to declare.

\section{Acknowledgements}

The authors gratefully acknowledge Ministry of Science and Technology, Taiwan, R.O.C (NSC 100-2113-M-033-002-MY3) and Chung Yuan Christian University for supporting the research work.

\section{References}

1 Y. Wang, K. S. Chen, J. Mishler, S. C. Cho and X. C. Adroher, Appl. Energy, 2011, 88, 981-1007.

2 C. H. Tsai, C. C. Wang, C. Y. Chang, C. H. Lin and Y. W. Chen-Yang, Int. J. Hydrogen Energy, 2014, 39, 1569615705.

3 T. Taner, J. Fundam. Renewable Energy Appl., 2015, 5, 163.

4 J. H. Wee, Renewable Sustainable Energy Rev., 2007, 11, 17201738.

5 W. M. Yan, C. Y. Hsueh, C. Y. Soong, F. Chen, C. H. Cheng and S. C. Mei, Int. J. Hydrogen Energy, 2007, 32, 4452-4458.

6 L. W. Zhang, S. R. Chae, Z. Hendren, J. S. Park and M. R. Wiesner, Chem. Eng. J., 2012, 204-206, 87-97.

7 C. H. Park, C. H. Lee, M. D. Guiver and Y. M. Lee, Prog. Polym. Sci., 2011, 36, 1443-1498.

8 Anonymous, Multi-year research, development and demonstration plan: planned program activities for 20052015, EIA Office of Oil and Gas DOE, Washington, 2007.

9 M. Mamlouk, P. Ocon and K. Scott, J. Power Sources, 2014, 245, 915-926.

10 N. Wehkamp, M. Breitwieser, A. Buchler, M. Klingele, R. Zengerleac and S. Thiele, RSC Adv., 2016, 6, 24261-24266.

11 N. Holmström, J. Ihonen, A. Lundblad and G. Lindbergh, Fuel Cells, 2007, 7(4), 306-313.

12 M. Prasanna, H. Y. Ha, E. A. Cho, S. A. Hong and I. H. Oh, J. Power Sources, 2004, 131, 147-154.

13 Y. W. Chen-Yang, T. F. Hung, J. Huang and F. L. Yang, J. Power Sources, 2007, 173, 183-188.

14 A. Arvay, E. Yli-Rantala, C. H. Liu, X. H. Peng, P. Koski, L. Cindrella, P. Kauranen, P. M. Wilde and A. M. Kannan, J. Power Sources, 2012, 213, 317-337.

15 S. Park, J. W. Lee and B. N. Popov, Int. J. Hydrogen Energy, 2012, 37, 5850-5865.

16 L. R. Jordan, A. K. Shukla, T. Behrsing, N. R. Avery, B. C. Muddle and M. Forsyth, J. Power Sources, 2000, 86, 250-254. 
17 L. Cindrella, A. M. Kannan, J. F. Lina, K. Saminathan, Y. Ho, C. W. Lind and J. Wertz, J. Power Sources, 2009, 194, 146-160.

18 T. F. Hung, J. Huang, H. J. Chuang, S. H. Bai, Y. J. Lai and Y. W. Chen-Yang, J. Power Sources, 2008, 184, 165-171.

19 H. Tang, S. Wang, M. Pan and R. Yuan, J. Power Sources, 2007, 166, 41-46.

20 L. Cindrella, A. M. Kannan, R. Ahmad and M. Thommes, Int. J. Hydrogen Energy, 2009, 34, 6377-6383.

21 A. El-kharouf, Understanding GDL Properties And Performance In Polymer, University of Birmingham Research Archive, 2010.

22 X. P. Tao, Y. J. Xiang, W. K. Sai, Z. Zhen and S. P. Wen, Chin. Sci. Bull., 2012, 57, 2948-2955.

23 A. Iwan, M. Malinowski and G. Pasciak, Renewable Sustainable Energy Rev., 2015, 49, 954-967.

24 K. S. Novoselov, A. K. Geim and S. V. Morozov, Science, 2004, 306, 666-669.

25 C. Yun, Y. Feng, T. Qiu, J. Yang, X. Li and L. Yu, Ceram. Int., 2015, 41, 8643-8649.

26 A. M. I. Trefilov, A. Tiliakos, E. C. Serban, C. Ceaus, S. M. Iordache, S. Voinea and A. Balan, Int. J. Hydrogen Energy, 2017, 42, 10448-10454.

27 A. T. Najafabadi, M. J. Leeuwner, D. P. Wilkinson and E. L. Gyenge, ChemSusChem, 2016, 9, 1689-1697.

28 H. L. Lin, T. L. Yu, K. S. Shen and L. N. Huang, J. Membr. Sci., 2005, 237, 1-7.

29 C. Han, I. T. Kim, H. J. Sun, G. Park, J. J. Lee, H. K. Lee and J. Shim, Int. J. Electrochem. Sci., 2012, 7, 8627-8636.
30 M. S. Ismail, A. Hassanpour, D. B. Ingham, L. Ma and M. Pourkashanian, Fuel Cells, 2012, 12(3), 391-397.

31 C. Lee, Science, 2008, 321(5887), 385-388.

32 S. K. Singh and S. S. Pawar, IJSRET, 2014, 3(7), 1050-1053.

33 A. Sveshnikova, K. Abrosimov, A. Khayrullina and A. Ustinov, J. Renewable Sustainable Energy, 2017, 9, 044301.

34 J. Bang, H. S. Kim, D. H. Lee and K. Min, Journal of Mechanical Science and Technology, 2008, 22, 1602-1611.

35 N. Ahmadi, S. Rezazadeh, A. Dadvand and I. Mirzaee, Period. Polytech., Chem. Eng., 2018, 62(1), 97-105.

36 M. Pérez-Page and V. Pérez-Herranz, Int. J. Electrochem. Sci., 2011, 6, 492-505.

37 B. Balasubramanian, F. Barbir, B. Byron, S. Nomikos and M. Stone, Fuel Chemistry Division Preprints, 2002, 47(2), 612.

38 S. Shahgaldi and J. Hamelin, Energy Convers. Manage., 2015, 103, 681-690.

39 S. M. Kim, C. Y. Ahn, Y. H. Cho, S. Kim, W. Hwang, S. Jang, S. Shin, G. Lee, Y. E. Sung and M. Choi, Sci. Rep., 2016, 6, 26503.

40 M. J. Kim, O. H. Kim, I. S. Park, Y. H. Cho and Y. E. Sung, Electrocatalysis, 2018, 9, 352.

41 T. F. Hung, S. H. Liao, C. Y. Li and Y. W. Chen-Yanga, J. Power Sources, 2011, 196, 126-132.

42 C. H. Tsai, F. L. Yang, C. H. Chang and Y. W. Chen-Yang, Int. J. Hydrogen Energy, 2012, 37, 7669-7676.

43 M. Amjadi, S. Rowshanzamir, S. J. Peighambardoust and S. Sedghi, J. Power Sources, 2012, 210, 350-357.

44 M. A. Navarra, C. Abbati, F. Croce and B. Scrosati, Fuel Cells, 2009, 09(3), 222-225. 